\title{
The Effect of Evaluation on Teacher Performance
}

\section{$B Y$ ERIC S. TAYLOR AND JOHN H. TYLER}

\section{Web Appendix}
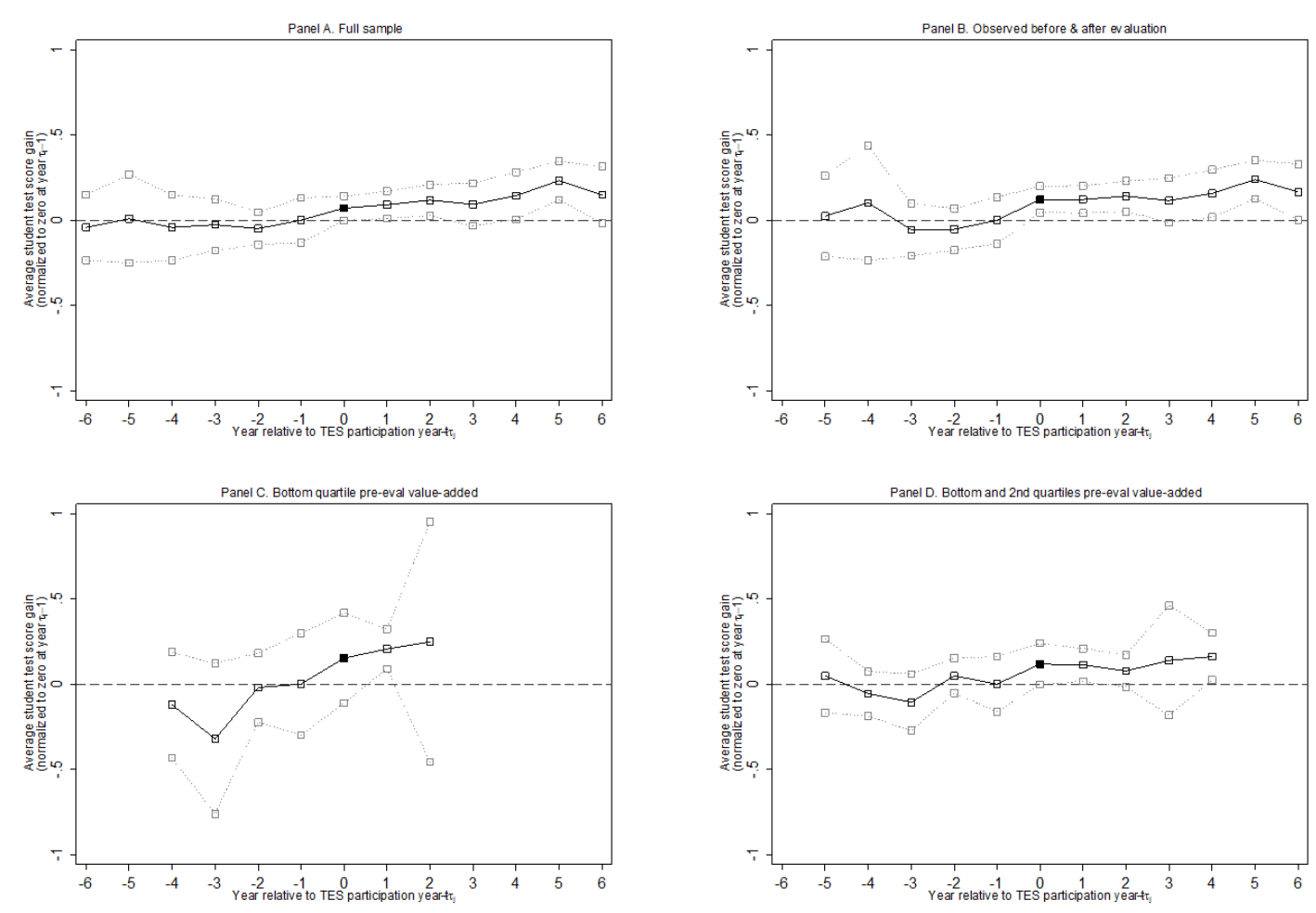

FIGURE 1A: TEACHER PERFORMANCE RELATIVE TO YEAR OF EVALUATION FOR SUBSAMPLES

Notes: Dotted lines represent 95\% confidence interval. 
Table A1: Reading achievement effects, and estimates by type of teaching assignment

\begin{tabular}{|c|c|c|c|c|c|}
\hline & & & & \multicolumn{2}{|c|}{ Math achievement } \\
\hline & \multicolumn{3}{|c|}{ Reading achievement } & $\begin{array}{c}\text { Teach math } \\
\text { only }\end{array}$ & $\begin{array}{l}\text { Teach math } \\
\text { and reading }\end{array}$ \\
\hline & (1) & (2) & (3) & (4) & $(5)$ \\
\hline \multicolumn{6}{|c|}{$\begin{array}{l}\text { School year relative to year of TES evaluation } \\
\text { (all other years prior omitted) }\end{array}$} \\
\hline Year of evaluation $(\mathrm{t}=\mathrm{\tau})$ & $\begin{array}{l}-0.009 \\
(0.036)\end{array}$ & $\begin{array}{l}-0.006 \\
(0.036)\end{array}$ & $\begin{array}{r}0.012 \\
(0.050)\end{array}$ & $\begin{array}{c}0.068 \\
(0.046)\end{array}$ & $\begin{array}{c}0.021 \\
(0.087)\end{array}$ \\
\hline All years after $(\mathrm{t}>\tau)$ & $\begin{array}{l}-0.050 \\
(0.038)\end{array}$ & $\begin{array}{l}-0.047 \\
(0.038)\end{array}$ & $\begin{array}{l}-0.051 \\
(0.058)\end{array}$ & $\begin{array}{l}0.114^{*} \\
(0.051)\end{array}$ & $\begin{array}{c}0.039 \\
(0.083)\end{array}$ \\
\hline Teacher experience quadratic & $\mathrm{Y}$ & & & Y & $\mathrm{Y}$ \\
\hline Student covariates & $\mathrm{Y}$ & $\mathrm{Y}$ & & $\mathrm{Y}$ & $\mathrm{Y}$ \\
\hline Teacher clusters & 144 & 144 & 144 & 50 & 55 \\
\hline Student-year observations & 18030 & 18030 & 18030 & 9597 & 4734 \\
\hline
\end{tabular}

Note: Each column reports a separate teacher fixed effects estimation of student standardized (by grade and year) test score as a function of grade-by-year fixed effects, and the indicated covariates. Student covariates as described in table 3 note. Clustered (teacher) standard errors in parentheses.

$* *$ indicates $\mathrm{p}<0.01, * \mathrm{p}<0.05$, and $+\mathrm{p}<0.10$. 


\begin{tabular}{lcc}
\hline \hline & & \\
& & $(1)$ \\
School year relative to year of TES evaluation & & \\
(year immediately prior, $\mathrm{t}=\tau-1$, omitted) & & \\
Six years prior $(\mathrm{t}=\tau-6)$ & -0.067 & 0.094 \\
& $(0.100)$ & $(0.166)$ \\
Five years prior & 0.0109 & -0.034 \\
& $(0.116)$ & $(0.120)$ \\
Four years prior & -0.009 & -0.031 \\
& $(0.092)$ & $(0.124)$ \\
Three years prior & -0.010 & -0.033 \\
& $(0.084)$ & $(0.139)$ \\
Two years prior & -0.051 & -0.044 \\
Year of evaluation $(\mathrm{t}=\tau)$ & $(0.048)$ & $(0.065)$ \\
First year after $(\mathrm{t}=\tau+1)$ & 0.051 & $0.077+$ \\
Second year after & $(0.038)$ & $(0.044)$ \\
Third year after & $0.081 *$ & $0.105 *$ \\
Fourth year after & $(0.037)$ & $(0.044)$ \\
& $0.125 * *$ & $0.137 * *$ \\
Fifth year after & $(0.040)$ & $(0.051)$ \\
& 0.111 & $0.174 *$ \\
& $(0.073)$ & $(0.080)$ \\
& $0.143 * *$ & $0.264 * *$ \\
& $(0.052)$ & $(0.070)$ \\
& $0.230 * *$ & $0.371^{* *}$ \\
& $(0.041)$ & $(0.067)$ \\
& $0.139 *$ & $0.307 * *$ \\
& $(0.065)$ & $(0.062)$
\end{tabular}

Teacher experience quadratic

Student covariates

Note: Each column reports a separate teacher fixed effects estimation of student standardized (by grade and year) math test score as a function of grade-by-year fixed effects, and the indicated covariates. Student covariates as described in table 3 note. Clustered (teacher) standard errors in parentheses. In each case the sample is comprised of 105 teachers and 14,331 student-by-year observations.

$* *$ indicates $\mathrm{p}<0.01, * \mathrm{p}<0.05$, and $+\mathrm{p}<0.10$. 